\title{
The application of ultrasound examination in the treatment of Acute Sinusitis. Comparing X-ray to ultrasound of paranasal sinuses
}

\author{
Al. Valkov', G. Nikolov',B. Duhlenski', Tvs. Stoyanov', Tsv. Mladenov', \\ M. Yildiz', Kr. Atanasova', St. Mirchev', Kr. Valcvheva² \\ 'Clinic of ENT and Maxillofacial surgery UMHAT „D-r Georgi Stransky" - Pleven, Bulgaria \\ ${ }^{2}$ Clinic of ophthalmology UMHAT „D-r Georgi Stransky“ - Pleven, Bulgaria
}

\begin{abstract}
Resume
Background: Acute rhinosinusitis (ARS) is one of the most common diseases encountered in the out-patient setting. X-ray of the paranasal sinuses is the gold standard in the diagnosis of ARS with its accessibility and accuracy. Ultrasound examination of the paranasal sinuses is a noninvasive diagnostic method which is an acceptable alternative in the follow-up of the treatment.

Aim: To present the application of portable sinuson in the out-patient setting on patients with ARS and its effectiveness.

Materials and Methods: 22 patients $(12 \mathrm{~m} / 10 \mathrm{f})$ underwent X-ray of the paranasal sinuses, which resulted in ARS. The results of the X-rays were compared with the ultrasound examination of the paranasal sinuses with a portable sinuson (SINUSULTRA ULTRAMAX). The examination was repeated on the 1-st and 2-nd week and 2 months after the treatment for ARS. Results: From the 22 patients -10 were with unilateral maxillary sinusitis, 7 with bilateral sinusitis and 5 with pansinusitis. $5(22 \%)$ false positives were registered from the ultrasound examination on the initial check-up, $3(13 \%)$ false negatives were registered on the follow-ups on the 1-st and the 2-nd week, 2 (9\%) false negatives were registered 2 months after the treatment for ARS.

Conclusion: Ultrasound examination of the paranasal sinuses is a noninvasive, painless, fast and with an adequate degree of accuracy. It is a good alternative for children and pregnant women. Ultrasound examination can be applied in the follow-up of ARS.
\end{abstract}

Key words: acute rhinosiuitis, ultrasound, follow-up

\section{Background}

Infections of the upper airways are the most common pathology. They are the most often associated with out-patient consultation by general practitioners and otorhinolaryngologist. It is normal for adults to have 4-5 episodes of some kind of upper airway infection per year, while for children, 10 episodes per year is considered common. Often acute rhinitis is complicated with infection of the paranasal sinuses. According to National Ambulatory Medical Care Survey acute rhinosinusitis is the 5-th most common cause for the prescription of antibiotics in the out-patient setting. There is a chance for bacterial superinfection of acute viral rhinosinusitis in $0.2-2 \%{ }^{[1]}$

Diagnosing acute rhinosinusitis (ARS) is often difficult solely based on symptoms and anterior rhinoscopy. Symptoms such as (rhinorrhea, nasal obstruction, headache, hyposmia or anosmia etc) can also be associated with uncomplicated infection of the upper airways. X-ray of the paranasal sinuses is considered the gold standard in the diagnosis of ARS in the out-patient setting, due to its accuracy and accessibility. In order to perform a proper follow-up at least two consecutive X-rays should be performed. This is associated with an increased radiation exposure for the patient. Due to this often the follow-up X-ray is not performed (especially for pregnant women and children) which leads to imprecise treatment and chronic rhinosinusitis (CRS). Ultrasound of the paranasal sinuses is a non-inva- 
sive, painless and mostly overlooked method in the diagnosis of ARS. It is not associated with radiation exposure and is easy to perform with the proper equipment. We have made it the aim of our study to compare the accuracy of ultrasound to X-ray of the paranasal sinuses in the diagnosis and follow-up of ARS in the out-patient setting.

\section{Aim}

The application of ultrasound diagnosis of the paranasal sinuses in the-out-patient setting. To compare the accuracy of ultrasound to $\mathrm{X}=$ ray of the paranasal sinuses in the diagnoses of ARS.

\section{Materials and Methods}

22 (12 male and 10 female) patients were selected after being diagnosed with ARS by X-ray of the paranasal sinuses. All the patients were diagnosed and observed in an out-patient ENT setting. Most patients were referred by General Practitioners, while 3 were referred by an ophthalmologist. On the initial check-up of the patient an X-ray of the paranasal sinuses is carried out. If there are radiological data suggesting ARS the patient is included in the study and an ultrasound of the paranasal sinuses is performed. The patient is treated according to the international guidelines for treatment of acute rhinosinusitis. The follow-up is carried out on the 1-st and 2-nd week and 2 months after treatment. Informed consent was obtained from all patients included in the study. In our cohort we have included 10 patients with unilateral sinusitis, 7 with bilateral and 5 with pansinusitis. Ultrasound was performed on the maxillary and frontal sinuses. We used the portable sinuzon (SINUSULTRA ULTRAMAX) - working frequency $3 \mathrm{MHz}$, diameter of the probe $8 \mathrm{~mm}$, pulse rate $300 \mathrm{~Hz}$. The echographic scale for maxillary sinus is $8 \mathrm{~cm}$ and for frontal $-4 \mathrm{~cm}$. Ultrasound diagnosis was made when there were concrete radiological features for ARS from the X-ray (Opacification of the sinuses, gas-fluid level). The results from the ultrasound diagnosis of the maxillary sinus were interpreted as follows: Echo $\leq 1.5$-Normal sinus; Echo $=2 \mathrm{~cm}$ - thickening of the mucosa; Echo from 4-5 cm fluid in the sinus; Echo with a double peak - Cyst of the sinus. For the frontal sinus - Echo $\leq 1 \mathrm{~cm}-$ normal sinus; Echo from 1-3 - Fluid in the sinus.

\section{Results}

On the initial checkup (first visit) all 22 patients had ARS diagnosed by X-ray of the paranasal sinuses. There were $5(22.7 \%)$ false negative results from the ultrasound examination which gives us an overall accuracy of $81.4 \%$. During the follow-up on 1-st and 2-nd week, there were both times 3 false negatives - accuracy of $88 \%$. For the follow-up 2 months post treatment out of 22 patients only 16 came for check-up. All of them were confirmed healthy by X-ray of paranasal sinuses. There were $2(12.5 \%)$ false positive results - accuracy of $88 \%$.

\section{Conclusion}

In the out-patient setting diagnosis of ARS without imaging is hard due to the unspecific presentation which is hard to differentiate from an acute viral rhinitis. Other contributing factors such as allergic rhinitis and pregnancy in women also muddy the waters in the diagnosis of ARS. This often leads to imprecise treatment which can lead to the exacerbation of the disease process. In the different studies, the accuracy of ultrasound diagnosis is between $32-99 \%$. In our study the accuracy is between $81-88 \%$. Although the number of patients included in our study is small, the results are promising and should be explored further. In our opinion ultrasound examination of paranasal sinuses when available is an acceptable alternative to X-ray of paranasal sinuses in the out-patient setting. This method is non-invasive, painless, fast and doesn't involve radiation exposure to the patient. It can be performed on children above 3 years and pregnant women. It can be applied in the follow-up of the treatment of ARS

\section{References}

1. Куцаров А. Остър риносинуит. Мединфо 2008; бр. 3.

2. Вичева Д. Ринити. 2004 г.

3. Landman MD Ultrasound screening for sinus disease. Otolaryngol Head Neck Surg. 1986;94157- 164Google Scholar

4. Rohr ASSpector SLSiegel SCKatz RMRachelefsky GS Correlation between A-mode ultrasound and radiography in the diagnosis of maxillary sinusitis. J Allergy Clin Immunol. 1986;7858- 61

5. Ц Цветков В., Златанов Хр., Колев, Кукушев Г. Ендоскопската синус хирургия FESS - златният стандарт при лечението на нарушеното носно дишане и заболяванията на околоносните кухини. Мединфо 2011, бр. 3

6. Landman MD Ultrasound screening for sinus disease. Otolaryngol Head Neck Surg. 1986;94157- 164 